\title{
Carbon Nanotube Graphoepitaxy: Highly Oriented Growth by Faceted Nanosteps
}

Ariel Ismach, David Kantorovich, and Ernesto Joselevich*

Department of Materials and Interfaces, Weizmann Institute of Science, Rehovot 76100, Israel.

E-mail: ernesto.joselevich@weizmann.ac.il

\section{Supporting Information}

\section{Definition of the miscut parameters}

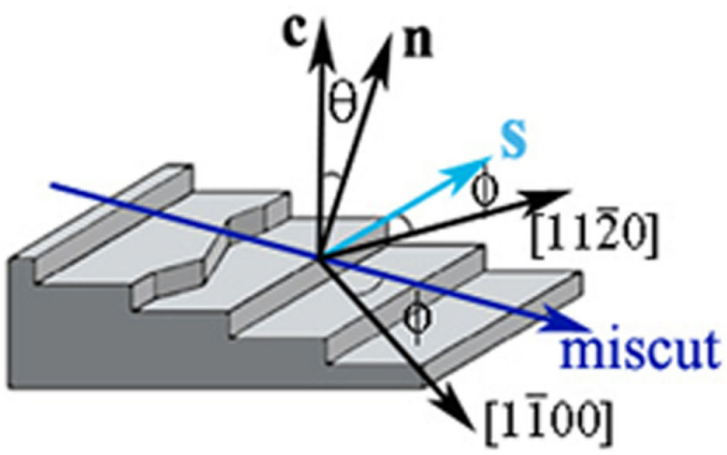

Figure S1. Miscut inclination and azimuth angles, $\theta$ and $\varphi$, respectively, and step vector s. The step vector is defined as $\mathbf{s}=\hat{\mathbf{c}} \times \hat{\mathbf{n}}$, where $\hat{\mathbf{c}}$ and $\hat{\mathbf{n}}$ are unit vectors normal to the C-plane and to the surface plane, respectively, so that $\mathbf{s}$ is parallel to the steps, descending to the right.

\section{Additional AFM images}
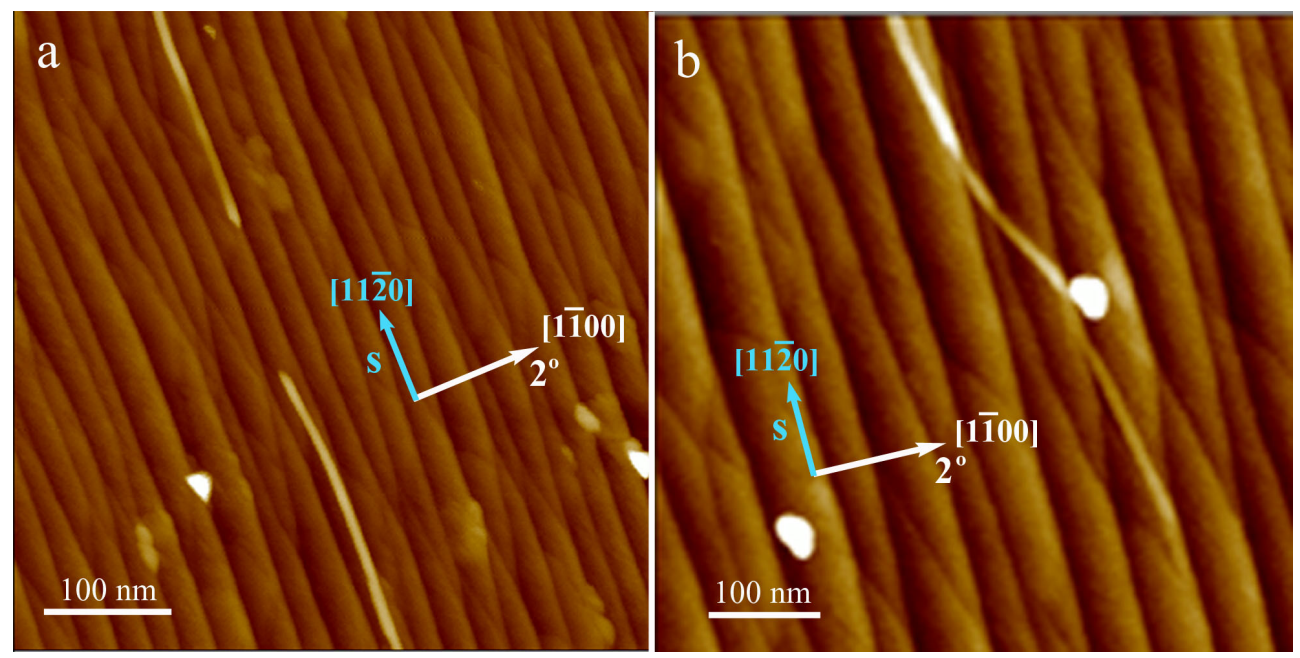

Figure S2. Topographic AFM images showing SWNTs competitively aligned by the nanoscopic and atomic steps present on certain surfaces. (a) The upper nanotube runs along a nanostep, while the lower one jumped onto an unbunched atomic step remaining in the middle of a terrace. (b) A SWNT jumps from one nanostep to another along unbunched atomic steps. 

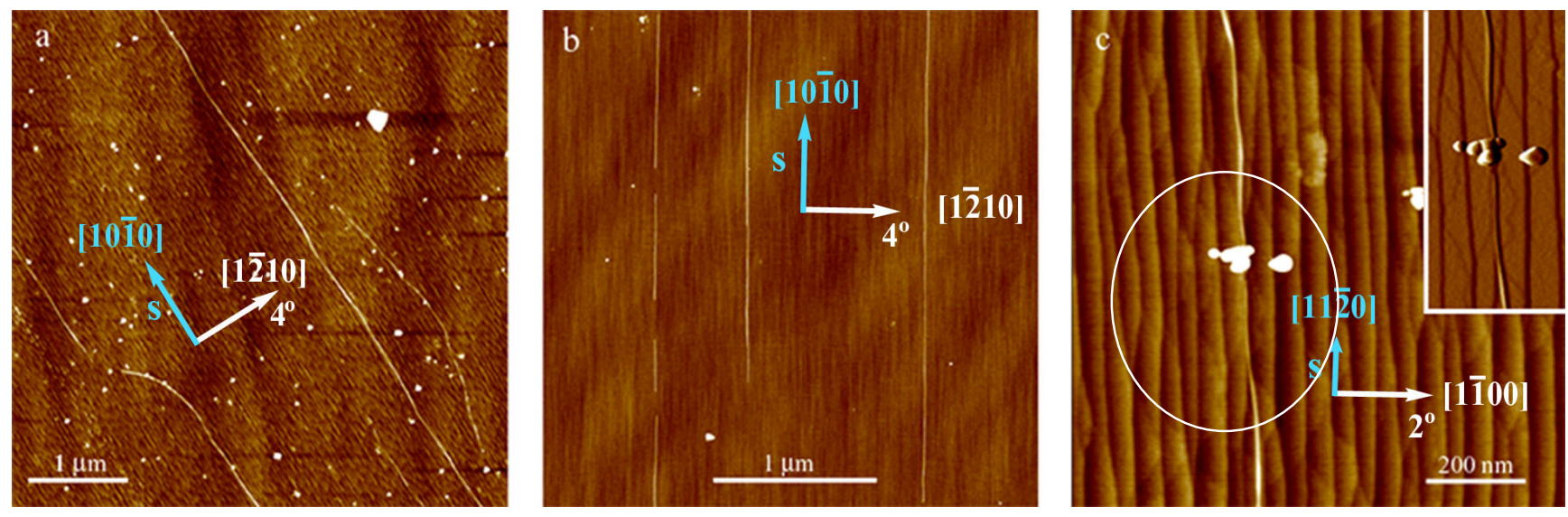

Figure S3. AFM images of selected samples showing different phenomena. (a) and (b) show two samples with similar miscut inclination and orientation, but different degrees of faceting (Figures $1 \mathrm{i}$ and 1g, respectively), leading to sawtooth-shaped or straight nanostep morphologies, respectively. (b). (c) Certain nanotubes can hide or be partly eclipsed by the nanosteps, giving the appearance of a dashed line, at a larger scale. The inset shows the amplitude image where the SWNT is hiding along the step.
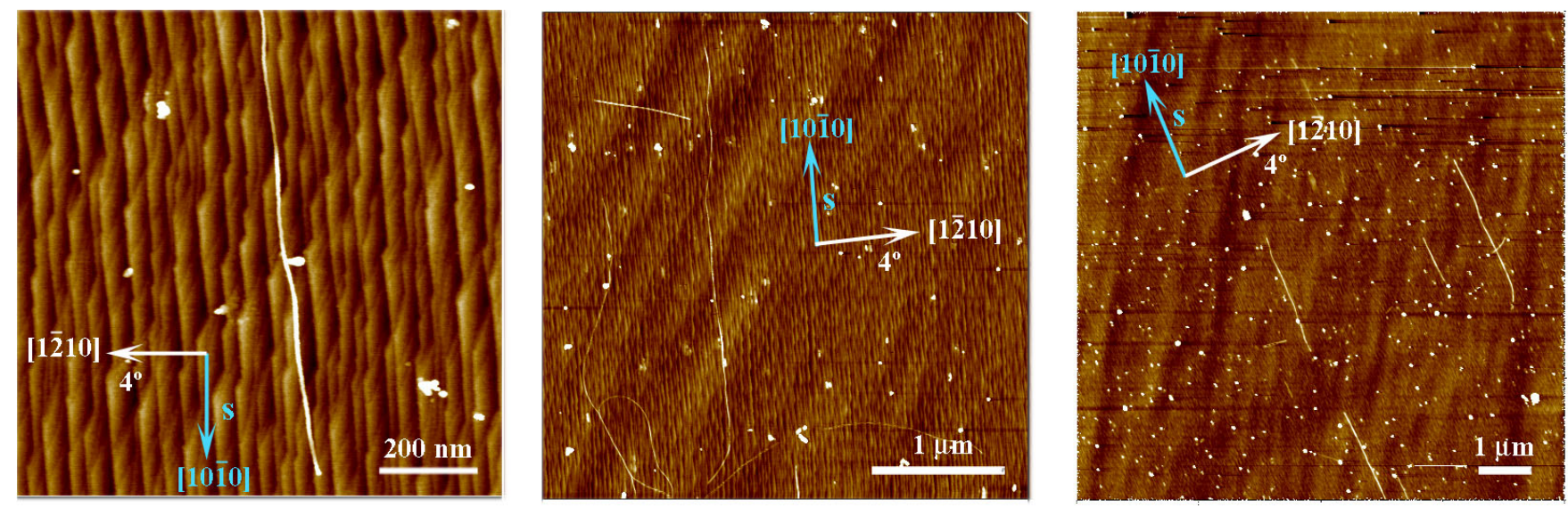

Figure S4. AFM images of additional samples displaying wavy SWNTs loosely conformal to sawtoothshaped faceted nanosteps. These samples exhibit a lower density and degree of alignment, which may be attributed to a weaker SWNT-nanostep interaction. 


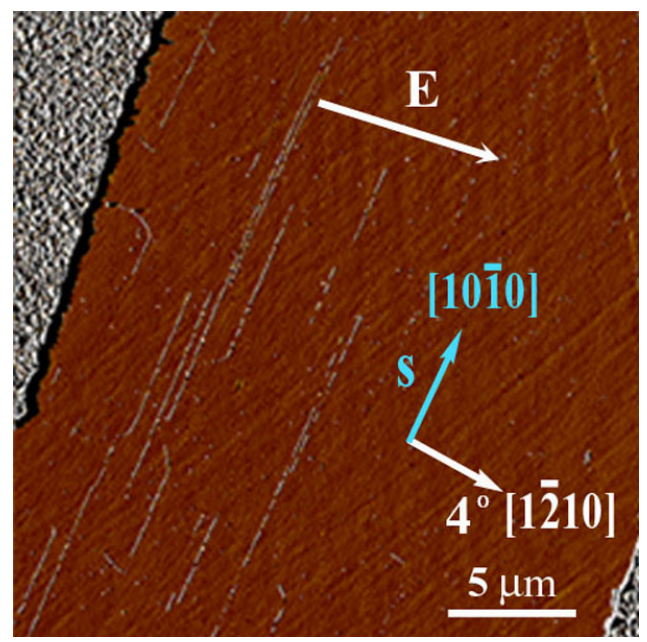

Figure S5. AFM image of aligned SWNTs along the nanosteps, s vector. During the CVD an external electric field (E, $2 \times 10^{6} \mathrm{~V} / \mathrm{m}$ ) was applied closely perpendicular to the s vector as shown in the image. However, the electric field had practically no effect on the growth direction.

\section{Methods}

C-plane sapphire wafers were purchased from Gavish Industrial \& Materials LTD., Omer, Israel (one side polished), with miscut inclination angles of $2^{\circ}$ and $4^{\circ}$ towards the [1100] and [1210] directions. The material was received first as an ingot. The lattice orientation was identified by a backreflection $\mathrm{x}$-ray diffraction $(\mathrm{XRD}) \operatorname{method}^{5}$ (see Figure S6 showing a XRD pattern from a low-miscut $\alpha$ $\mathrm{Al}_{2} \mathrm{O}_{3}$ (0001) sample). After marking the exact directions, the ingots were sent back to Gavish Company for cutting according to the specifications described above, and mechanical polishing. Samples that were chemically-mechanically polished (Marketech International) gave poorer yield and alignment. X-ray Photoelectron Spectroscopy (XPS) showed different amounts of surface impurities (e.g. Na, Mg, Ca), which could arise from the chemical treatment, and affect the nanotube growth process. The miscut inclination and azimuth angles were determined by X-ray diffraction, by an asymmetric doubleexposure back-reflection method ${ }^{5}$, Figure S7. The X-rays were produced by an Elliot GX6 rotating anode generator operating at $1.2 \mathrm{KW}$ and producing $\mathrm{Cu}$ radiation with a $200 \mu \mathrm{m}$ focus, with smallest wavelength of $0.41 \AA$ through $\mathrm{k}_{\alpha}(1.54 \AA)$ and residual lower intensities of larger wavelengths. The 
sample, placed perpendicular to the X-ray beam, was first exposed to back reflections for $2 \mathrm{hrs,} \mathrm{and} \mathrm{then}$ rotated by $180^{\circ}$ for a second exposure of $1 \mathrm{hr}$. In most cases a back reflection Laue pattern would have a larger number of reflections, which would be difficult to interpret. In order to reduce the number of spots and thus facilitate the recognition of both patterns, from the first and second exposures, a nickel filter was used to cut off the $\mathrm{k}_{\beta} 1.39 \AA$ and shorter radiations. We know that $80 \%$ of the reflections are from radiation wavelengths between 1.5-1.8 $\AA$. The miscut angle $\theta$ was measured by a systematic and an approximated method, both giving the same results: (i) The spots of the XRD patterns were placed on an appropriate Greninger chart in order to read the angular relations on the back reflection film, and then the spots were plotted on a stereographic projection to measure the miscut inclination and azimuth; (ii) The distance between the centers of the first and second patterns was measured and defined as $2 R$. Then the miscut inclinations given by $\theta=\tan ^{-1}(\mathrm{R} / \mathrm{L}) / 2$, where $\mathrm{L}=3 \mathrm{~cm}$ is the distance between the sample and the X-ray sensitive film $(7 \times 7 \mathrm{~cm})$ on which the Laue patterns were recorded.

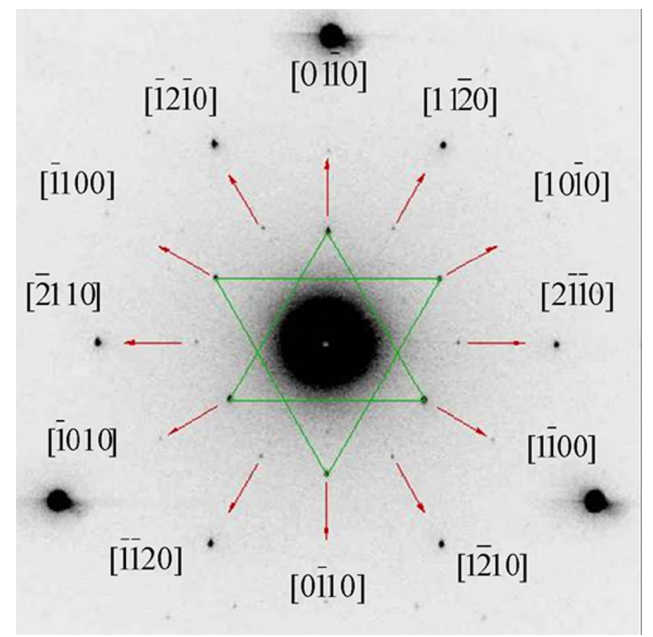

Figure S6. Back reflaction XRD pattern of a low-miscut $\alpha-\mathrm{Al}_{2} \mathrm{O}_{3}(0001)$ surface, showing the different low-index lattice directions. 


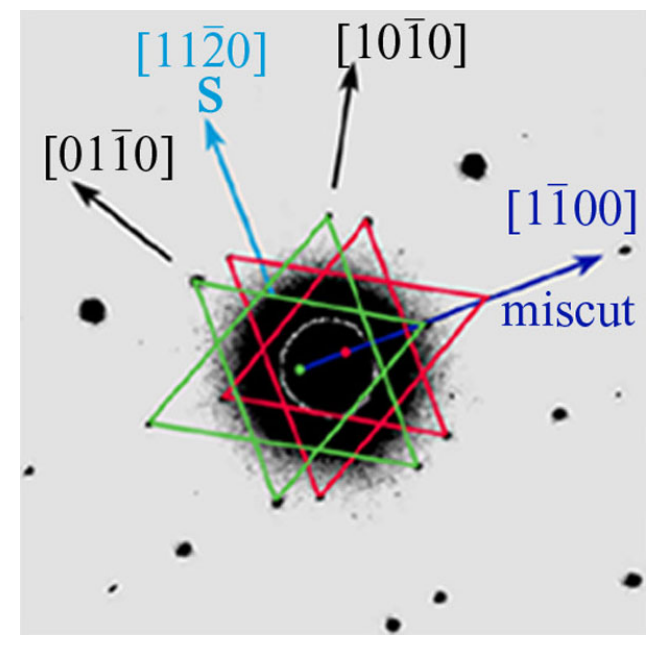

Figure S7. Asymetric double exposure back reflection XRD pattern of a vicinal $\alpha-\mathrm{Al}_{2} \mathrm{O}_{3}(0001)$ sample with miscut towards [1100] direction and miscut inclination angle of $\theta=2.1 \pm 0.1^{\circ}$.

The $5 \mathrm{~cm}$ wafers were cut in pieces of about $1 \times 1 \mathrm{~cm}$, and cleaned by sonication in acetone for 15 min, followed by rinsing in isopropanol and deionized water, blow dried and annealed at $1100^{\circ} \mathrm{C}$ for 5 to10 hours in air.

Single-wall carbon nanotubes (SWNTs) were grown by catalytic chemical vapor deposition (CVD), using ferritin protein as a precursor for monodisperse Fe nanoparticle catalyst, as previously reported ${ }^{2,5}$. Substrates were first oxidized by oxygen plasma (March Plasmod GCM 200, 1-3 min, with 1 sccm of $\mathrm{O}_{2}$ ), a ferritin solution of $0.1 \mathrm{~g} / \mathrm{L}$ (horse spleen ferritin, Sigma) was deposited on the samples for $10 \mathrm{~min}$, washed with deionizied water and blow dried, followed by a second plasma oxidation step, to eliminate all the organic matter. Finally, the CVD was carried out at $800^{\circ} \mathrm{C}$ for 10 min with a mixture of $60 \% \mathrm{Ar}$ (99.998\%, Oxygen \& Argon Industries, Israel), 40\% $\mathrm{H}_{2}$ (99.999\%, Gordon Gas, Israel) and 0.2\% ethylene $(99.9 \%$, Gordon Gas, Israel) at 1 atm and flow rate of $1 \mathrm{~L} / \mathrm{min}$. For the CVD experiments under electric field, platinum electrodes were patterned on the sapphire substrates, by a standard photolithography (photoresist Microposit S1805, Shipley), followed by electron beam evaporation (Edwards Auto 306) of $10 \mathrm{~nm}$ titanium (99.99\%, Holland Moran Ltd., Israel) and 90nm platinum (99.99\%, Holland Moran Ltd. Israel), while cooled with liquid nitrogen. The lithographic electrodes 
were connected with 4523AD Kulicke \& Soffa wire bonder, to external electrodes. A DC voltage of 50 $\mathrm{V}$ was applied between the electrodes (separation $25 \mu \mathrm{m}$ ) during CVD.

Atomic force microscopy (AFM) characterization of the SWNT and annealed sapphire was carried out in air tapping mode (Veeco, Multimode Nanoscope IV), using $70 \mathrm{kHz}$ etched Si probes (FESP, Nanoprobes). Field-emission scanning electron microscopy (FE-SEM) was done with a LEO Supra $55 \mathrm{VP}$, in ultra high vacuum using relatively low voltages $(0.3-1 \mathrm{kV})$ to minimize charging. 